\title{
REVIEW
}

Open Access

\section{Managing head and neck cancer patients during the COVID-19 pandemic: the experience of a tertiary referral center in southern Italy}

Francesco Longo', Eleonora M. C. Trecca ${ }^{1,2^{*}}$ (D, Aurelio D'Ecclesia', Chiara Copelli ${ }^{3}$, Karim Tewfik ${ }^{1}$, Alfonso Manfuso ${ }^{1}$, Nicola Pederneschi ${ }^{1}$, Annalisa Mastromatteo ${ }^{1}$, Matteo Aldo Russo ${ }^{1}$, Antonio Pansini ${ }^{4}$, Luca M. Lacerenza',

Pier Gerardo Marano ${ }^{1,2}$ and Lazzaro Cassano ${ }^{1}$

\begin{abstract}
Background: The medical community has been deeply involved in fighting the Coronavirus disease 2019 (COVID19) pandemic and, as a consequence, the care of non-COVID-19 patients has been impacted. However, the treatment of head and neck cancer patients is not deferrable, and an integrated strategy is required. The aim of the current article is to present the experience in the management of head and neck patients during the COVID-19 pandemic at the research hospital "Casa Sollievo della Sofferenza". This review contains replicable and widely usable instructions on how to avoid delays in the diagnosis and treatment of head and neck tumors and to ensure a gradual return to elective procedures.

Main text: The Head and Neck Department of the research hospital "Casa Sollievo della Sofferenza" includes an Otolaryngology and a Maxillofacial Surgery Unit, both of which deal with the diagnosis and treatment of benign and malignant pathologies of the head and neck, as well as urgent/emergent consultations and surgical procedures that necessitate time sensitive operative management, such as cochlear implantation (CI). Given these premises and the complexity of the Department, the "COVID-19 organizing protocol" of the research hospital "Casa Sollievo della Sofferenza" was divided into two phases in accordance with the different stages of the pandemic and the priority of treatment. Special attention was given to the medical surveillance of health care workers and hospitalized patients, to the organization of the outpatient clinic and the operating setting as well as to the implementation of telehealth systems.

(Continued on next page)
\end{abstract}

\footnotetext{
*Correspondence: eleonoramc.trecca@gmail.com; eleonora.trecca@unifg.it

'Department of Maxillofacial Surgery and Otolaryngology, IRCCS Casa

Sollievo della Sofferenza, Viale Cappuccini, 1, 71013 San Giovanni Rotondo

(Foggia), Italy

${ }^{2}$ Department of Otolaryngology- Head and Neck Surgery, University Hospital

of Foggia, Foggia, Italy

Full list of author information is available at the end of the article
}

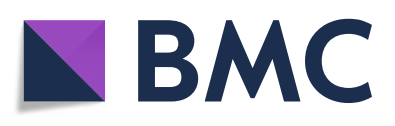

(- The Author(s). 2021 Open Access This article is licensed under a Creative Commons Attribution 4.0 International License, which permits use, sharing, adaptation, distribution and reproduction in any medium or format, as long as you give appropriate credit to the original author(s) and the source, provide a link to the Creative Commons licence, and indicate if changes were made. The images or other third party material in this article are included in the article's Creative Commons licence, unless indicated otherwise in a credit line to the material. If material is not included in the article's Creative Commons licence and your intended use is not permitted by statutory regulation or exceeds the permitted use, you will need to obtain permission directly from the copyright holder. To view a copy of this licence, visit http://creativecommons.org/licenses/by/4.0/. The Creative Commons Public Domain Dedication waiver (http://creativecommons.org/publicdomain/zero/1.0/) applies to the data made available in this article, unless otherwise stated in a credit line to the data. 
(Continued from previous page)

Conclusions: The COVID-19 pandemic is going to be a long-term situation with lasting effects on the public health and the entire society. Therefore, an efficient health care system has to adopt a double strategy: always being ready for a "new wave" of the pandemic and not forgetting non-COVID-19 patients, among whom head and neck cancer patients represent a priority. More than 1 year since the first outbreak in Wuhan, this review offers a unique and helpful perspective that incorporates awareness of the disease.

Keywords: Coronavirus disease, Critical care, Hospital administration, Ambulatory care, Primary health care, Otolaryngology, Maxillofacial surgery, Head and neck cancer, Infection

\section{Introduction}

Coronavirus Disease 2019 (COVID-19) is a global pandemic caused by the novel severe acute respiratory syndrome coronavirus 2 (SARS-CoV-2) infection, which is responsible for a wide range of mild to severe symptoms of the respiratory tract and multiple organ systems. In its most severe forms, the treatment of COVID-19 involves a multi-specialist approach and an integrated strategy [1]. Since the first cases in December 2019 in Wuhan, China, the medical community has been deeply involved in fighting this unprecedented pandemic and, as a consequence, the management of non-COVID-19 patients has been profoundly impacted. A survey conducted by the Italian Society of Otolaryngology-Head and Neck Surgery (SIOeChCF) reported a decrease in the number of elective otolaryngology (ORL) activities by $81.24 \%$ and a conversion of the Italian ORL departments in COVID-19 units in $22 \%$ of cases [2], while another study evidenced a reduction in the number of ORL emergency consultations by $80.8 \%$ [3]. Although the treatment of many Ear, Nose and Throat (ENT) conditions could be deferrable without any consequences, this is not the case in head and neck cancer patients whose care has to be prioritized during these difficult times [4]. Besides maintaining high-quality standards of treatment, the other main priority of regional cancer hubs has been the prevention of COVID-19 nosocomial infections. In addition to guaranteeing patients' safety, the protection of physicians and health professionals plays a role of primary importance in limiting the spread of the disease [5]. Otolaryngology-head and neck surgery (ORL-HNS) has been recognized as one of the specialties at highest risk since March 2020 [6] due to the symptoms of upper respiratory tract infections (URTI) which can be similar to the manifestations of COVID-19 (i.e. smell and taste dysfunction [7, 8], dysphonia [9]) and to the invasive nature of examinations (i.e. nasal endoscopy) and aerosol-generating procedures (AGPs) (i.e. functional endoscopic sinus surgery [10], tracheostomy [11], mastoidectomy [12], orthognathic surgery [13]). The international registry of Otolaryngologists-head and neck surgeons promoted by the Young Otolaryngologists-International Federation of Otolaryngologic
Societies (YO-IFOS) has identified 361 Otolaryngologists reporting COVID-19 at the time of publication in May 2020 and, unfortunately, this number has increased worldwide during the last months [14]. Another multicentric survey conducted in 23 Italian maxillofacial surgery departments found that $4 \%$ of maxillofacial surgeons and $22 \%$ of maxillofacial surgery residents tested positive for COVID-19 [15].

Given these premises, we would like to present our experience in the management of head and neck patients during the COVID-19 pandemic at the research hospital "Casa Sollievo della Sofferenza", which has been recognized as the best hospital of Southern Italy, according to the annual Newsweek report [16] and is located in one of the hardest hit provinces of the Puglia region. The main purpose of this review is to provide replicable and widely usable recommendations in order to avoid delays in the diagnosis and treatment of head and neck tumors, which can be life changing for the patients both in terms of mortality and quality of life, while ensuring a gradual return to elective procedures.

\section{The "COVID-19 organization protocol" at the research hospital "Casa Sollievo della Sofferenza"}

The "COVID-19 organization protocol" at the research hospital "Casa Sollievo della Sofferenza" was created in cooperation with the Preventive Medicine and Hygiene Section of the Institution and in accordance with the guidelines from the Higher Institute of Health (ISS).

The Head and Neck Department includes an Otolaryngology and a Maxillofacial Surgery Unit, both of which deal with the diagnosis and treatment of benign and malignant pathologies of the head and neck, such as benign and malignant tumors of the salivary glands, the oral cavity, the skin, the larynx, the paranasal sinuses and anterior skull base. Free flap surgery is executed by the Maxillofacial Surgeons for the reconstruction of complex head and neck defects and facial reanimation. The anterolateral thigh and radial forearm free flaps are the most commonly performed procedures at our institution, 50 and 49 flaps on average per year, respectively according to a recent case review [17]. Additionally, the Head and Neck Department performs urgent/emergent 
consultations (i.e., maxillofacial traumas, vertigo, inflammatory diseases, foreign bodies, airway obstructions, sudden hearing loss, epistaxis, etc.), which requires a high priority level within the hospital administration. Besides the ORL emergencies and cancer patients, the Head and Neck Department is also a referral center for other surgical procedures, such as cochlear implantation $(\mathrm{CI})$, that necessitate time sensitive operative management [18]. Given these premises and the complexity of the organization, an integrated strategy was requested in order to provide adequate treatment to every patient within an appropriate timeframe.

\section{Medical surveillance of health care workers and hospitalized patients during the COVID-19 pandemic}

The safety of health care workers plays a central role in guaranteeing the best possible treatments to patients. The Head and Neck Department currently employs one Director, 10 otolaryngologists, 6 maxillofacial surgeons, 3 resident physicians, 22 registered nurses, 4 speech therapists, 3 audiometric technicians, 10 healthcare operators and one health coordinator. The hospital requires health care workers to undergo nasal/oropharyngeal swabs for the detection of COVID-19 every month, in case of suspicion/contact with positive individuals, and for those returning after travelling outside the Puglia region. Specifically, molecular tests are the first choice at our hospital for the diagnosis of SARS-CoV-2 infection and the procedure comprises the execution of a double nasal/oropharyngeal swab. The nasal/oropharyngeal swabs are routinely performed by the otolaryngologists on patients/healthcare workers, and otolaryngologists are subjected to swabs for diagnosis, as well. The support of the ENT team is fundamental for minimizing the false negative rate among COVID-19 positive individuals [19].

Patients admitted to the Head and Neck Department have to test negative for COVID-19 in order to be hospitalized or must undergo a swab test, if referred for emergency ORL consultations by the Emergency Department. Patient visitors are routinely not allowed in the Department; exemptions are granted upon request for valid reasons. In case of undeferrable ENT conditions, such as dyspnea, hemorrhage, which require immediate treatment, without the possibility to perform a swab test, patients are considered positive and temporarily hospitalized in a distinct "COVID-19 gray area". This area has a dedicated patient room/operating room with separate entrances and negative pressures where suspected patients can receive the appropriate treatment and/or undergo the life-saving surgical procedure while waiting for the response of molecular test (usually 3-6h). In such cases, the health care personnel wear personal protective equipment (PPE) indicated by the guidelines of the SIOeChCF for tracheostomies [20]/surgical activities [21] which consists of FFP2 or preferably FFP3 mask, face shield, double gown, double gloves, double cap and shoe cover. This was the recent case of a patient (male; 53 years old) affected by a laryngeal malignant tumor classified as IVA according to American Joint Committee on Cancer (AJCC) Cancer Staging Manual (8th Edition), who firstly underwent a tracheostomy in the "COVID-19 gray operating room" and was subsequently hospitalized in the Head and Neck Department after testing negative. Two days after the tracheostomy, he could safely undergo a total laryngectomy with bilateral cervical lymphadenectomy without any delay in diagnosis and treatment.

Also, it is worth noting that head and neck cancer patients warrant additional surveillance during the COVID-19 pandemic as they are particularly susceptible to pulmonary infections and respiratory complications [22]. This was the recent case of a patient (male; 50 years old) hospitalized in the Head and Neck Department who underwent an open partial horizontal laryngectomy (OPHL) type IIa and developed post-operative pneumonia documented by computed tomography (CT). After excluding bacterial and fungal etiologies, a high level of suspicion for SARS-CoV-2 infection was raised and the patient was isolated while undergoing several tests in cooperation with the Internal Medicine Department. Besides blood tests for the differential diagnosis (i.e., Cytomegalovirus, Epstein-Barr virus), the patient underwent two other swab tests and serology testing for COVID-19 after 1 week, all of which were negative. Bronchoalveolar lavage (BAL) was performed to definitively exclude COVID-19 and the patient was able to stop isolation without any risks to the other patients and health workers.

Three registered nurses and one Otolaryngologist tested positive for COVID-19 thus far, while no hospitalized patients with COVID-19 have been identified. Therefore, it is possible that the health care workers who tested positive contracted the disease outside the hospital or in a location other than the Head and Neck Department. Certainly, this vigilant medical surveillance limited the spread of the disease and the occurrence of an outbreak so that even during the Italian lockdown, interruption to the activities of the Head and Neck Department or its conversion in COVID-19 unit, was never required.

\section{Guide to provide care during the COVID-19 pandemic} The "COVID-19 organizing protocol" at the research hospital "Casa Sollievo della Sofferenza" was divided into two phases in accordance with the different stages of the pandemic and the priority of treatment: 
- Phase 1 protocol (carried out from March 2020 to May 2020): The Head and Neck Department was active only for urgent/emergent surgical procedures and consultations, as well as for the management of undeferrable oncologic patients, including those scheduled for reconstructive procedures.

- Phase 2 protocol (from June 2020 to present): The Head and Neck department is gradually returning to the clinical and surgical routine of the pre-COVID19 era. Besides the procedures and consultations included in the "phase 1 protocol", the surgical procedures scheduled in this phase include at least one CI per week, as well as tympanomastoidectomy for chronic ear disease/cholesteatoma prioritizing cases with imminent complications, removal of benign tumors of head and neck, orthodontic-orthognathic surgery in disabled patients, functional endoscopic sinus surgery (FESS) including dacryocystorhinostomy (DCR) for inflammatory disease priority cases with impending complications, sleep surgery including transoral robotic surgery (TORS) priority patients affected by severe obstructive sleep apnea syndrome (OSAS) patients (apnea hypopnea index, AHI: > 30). Table 1 demonstrates the changes of head and neck pathologies presented by hospitalized patients at our department before and during the COVID-19 pandemic.
Additionally, the Head and Neck team designated two maxillofacial surgeons and two otolaryngologists to be in charge of consultations for the hospitalized COVID-19 patients, as well as for the tracheostomies in COVID-19 patients in the intensive care unit (ICU). Tracheostomies have been performed by the Head and Neck team in 23 COVID-19 patients thus far. However, according to the call to action from the European Laryngological Societies [23], the recommendation of our institution was to delay tracheostomy until the patient no longer requires ventilation in the prone position. Therefore, the main indication for tracheostomy was limited to COVID-19 patients with prolonged endotracheal intubation lasting more than 14-21 days. Patients discharged from the ICU are then scheduled for a strict ORL follow-up in cooperation with the Laryngologist in our department to monitor the potential occurrence of laryngotracheal complications (i.e., stenosis, granulomas, etc.).

Lastly, the pre-COVID-19 number of hospital beds was 34 and has been reduced by $50 \%$ during the pandemic. Therefore, the current number of beds in the Head and Neck Department is 16 plus one bed available for urgencies/emergencies. No more than one patient in a double room and two patients in a quadruple room are being hospitalized in phase 1 and 2 in order to guarantee adequate distance between individuals. One empty bed is left between one patient and another whenever

Table 1 Head and neck pathologies of hospitalized patients before and during the COVID-19 pandemic

\begin{tabular}{|c|c|c|c|}
\hline DISEASES & $\begin{array}{l}\text { Pre-COVID-19 } \\
\text { Time }^{\mathrm{a}}\end{array}$ & $\begin{array}{l}\text { COVID-19 } \\
\text { Time }^{\text {b }}\end{array}$ & $\begin{array}{l}\text { Percentage decrease } \\
\text { (\%) }\end{array}$ \\
\hline Adenoid hypertrophy/adenotonsillar hypertrophy & 108 & 4 & $-96.3 \%$ \\
\hline Airways obstruction (dyspnea) & 63 & 53 & $-15.9 \%$ \\
\hline Benign tumors of head and neck & 64 & 39 & $-39.1 \%$ \\
\hline Congenital cysts and fistulas of the neck & 4 & 0 & $-100 \%$ \\
\hline Diseases of the paranasal sinuses and tympanomastoid cavity requiring surgery & 116 & 32 & $-72.4 \%$ \\
\hline Dentofacial deformity & 66 & 31 & $-53.0 \%$ \\
\hline Epistaxis & 16 & 8 & $-50.0 \%$ \\
\hline Pharyngotonsillitis/ peritonsillar abscess & 23 & 11 & $-52.2 \%$ \\
\hline Malignant neoplasms of the head and neck & 58 & 56 & $-3.7 \%$ \\
\hline Maxillofacial trauma & 71 & 38 & $-46.5 \%$ \\
\hline Miscellanea & 373 & 170 & $-54.4 \%$ \\
\hline Nasal bone fractures & 36 & 12 & $-66.7 \%$ \\
\hline Nasal deformities/septal deviation & 33 & 0 & $-100 \%$ \\
\hline Otitis media & 27 & 7 & $-74.1 \%$ \\
\hline $\begin{array}{l}\text { Otitis media with effusion } \\
\text { (ear tube insertion) }\end{array}$ & 17 & 0 & $-100 \%$ \\
\hline Vertigo & 45 & 8 & $-82.2 \%$ \\
\hline
\end{tabular}

aPre-COVID-19 Time: March 1st to November 30th, 2019

${ }^{\mathrm{b}}$ COVID-19 Time: March 1st to November 30th, 2020 
possible or at least $2 \mathrm{~m}$ of space between beds. As an additional safe distancing measurement, patients' beds are placed diagonally [24].

\section{Organization of the outpatient clinic}

During the Italian lock-down, which limited people's movement and coincided with the "phase 1 protocol", the role of the telephone triage was fundamental. Dedicated personnel were in charge of ascertaining the absence of signs/symptoms of COVID-19 and establishing the urgency/priority of the visit. With the "phase 2 protocol", the activities of the outpatient clinic gradually returned back to the ordinary routine and patients on the waiting list were recalled. Twenty outpatients per day (Monday-Friday) were usually scheduled before the COVID-19 pandemic. The number of visits was reduced to 6 during the "phase 1 protocol" and almost returned to normal during the "phase 2 protocol" with 18 outpatients visited per day. Plexiglass protective barriers have been installed in order to prevent the spread of respiratory droplets and protect patients and personnel. According to the type and complexity of the visit, a waiting time ranging from 20 to $40 \mathrm{~min}$ is guaranteed between one patient and another in order to sanitize and aerate the room.

In accordance with the guidelines of the SIOeChCF [25], the health care workers admitted to the outpatient clinic should be limited to the essential (a nurse, an attending and a resident). The temperature check is done immediately upon arrival. Only the patient is admitted to the visit; visitors are not allowed, except for children and patients with disabilities. Physician and patient should maintain one-meter-distance in between each other during the interview. Empty seats should be left in the waiting room in order to ensure social distancing.

Both health care professionals and the patient should wash and / or sanitize their hands before the visit starts. Personnel should wear a disposable water-repellent gown, face shield, FFP2 or preferably FFP3 mask, cap and non-sterile gloves. During the visit it is important that the patient wears a surgical mask that should only be removed when necessary.

\section{Organization of the operating setting}

According to the guidelines of the SIOeChCF [21], patients have to undergo a swab test for the detection of SARS-CoV-2 infection within $48 \mathrm{~h}$ before surgery. When, for any reason, it is not possible to execute a swab test, the patient should be considered positive for COVID-19. When patients test negative, high-level PPE are not required, while in cases of positive or suspected patients, the personnel should be limited to the essential and wear double gowns, double gloves, shoe cover, double cap, FFP2 or possibly FFP3 mask covered by a surgical mask and face shield.

Negative pressures in the operating rooms are fundamental in limiting the diffusion of pathogens, including SARS-CoV-2.

The use of debriders, drills, powered instruments and electrocoagulation should be limited or avoided. When this is not possible, the use of high-level PPE together with COVID-19 patient screening is strongly recommended [26]. The same PPE are warranted for the team executing tracheostomies in COVID-19 patients. Further precautions are trying not to puncture the cuff of the tracheostomy tube and preferring cuffed non-fenestrated tubes.

Moreover, especially during these critical times, it is important for head and neck surgeons to work in cooperation with the ICU and check the availability of beds before performing any complex surgical procedures (i.e., TORS, free flap surgery, laryngectomy).

\section{Toward the health system of the future: implementing telehealth systems in the COVID-19 era}

In the last few years telehealth systems have become increasingly important and more and more sophisticated; likely, telehealth consultations are going to become ordinary in medicine in the near future and patients will be increasingly followed virtually. This implementation will help reduce the costs and inconvenience of travelling and clinics will be able to receive external technical support very quickly. Additionally, physicians and therapists will be able to provide care remotely and guarantee access to high-level services even to underserved and rural areas [27].

In the era of "social distancing", the potentialities of eHealth and telemedicine appear even more promising and our institution is also taking advantage of these systems. In fact, during the "phase 1 protocol", the CI team in the Head and Neck Department performed the first "remote fitting" [28] of a CI system. The implanted patient was a woman living outside of the Puglia region who underwent CI surgery in March 2020, prior to the Italian lockdown. Activation, mapping and measurements were performed by the $\mathrm{CI}$ team during a telehealth session preventing the patient from having to travel a distance during the critical phase of lockdown. In-person visits gradually started to be scheduled again with the activation of "phase 2 protocol", but the" remote fitting" system was successfully tested and is ready for an eventual return to the "phase 1 protocol".

Additionally, telehealth consultations played a central role in the management of head and neck cancer patients. The Department supported a campaign promoted by the Italian Association of Head and Neck Oncology (AIOCC) [29] to raise awareness for head and neck 
cancer tumors. Patients were invited to fill out an online questionnaire and subjects at risk were automatically scheduled for a free telehealth consultation and/or an in-person visit.

\section{Discussion and conclusions}

The fear of this "new COVID-19 era" should not determine a "new cancer era". Head and neck surgeons know how crucial early diagnosis is in oncological patients, as it implies higher probability of treatment success. Therefore, when counseling head and neck cancer patients, the take home message of this first year of the COVID19 pandemic should be "the sooner, the better". From our experience, one of the greatest difficulties was the cancer screening, as patients tend to seek medical attention at a more advanced disease stage or when they have already developed life-threatening complications. In our opinion, the fear of acquiring a nosocomial COVID-19 infection together with the inappropriate organization of many hospitals played a central role in this delay. Conversely, the oncological patients already scheduled for surgical operations experienced a shorter waiting time than the pre-COVID-19 era due to the cancellation/reduction of elective procedures [30].

One year since the first outbreak in Wuhan, China, we now have a clear understanding that the COVID-19 pandemic is going to be a long-term situation with lasting effects on public health and the entire society [31]. Therefore, an efficient health care system has to adopt a double strategy in this historical moment: always being ready for a surge of COVID-19 cases, while not forgetting non-COVID-19 patients, among whom, head and neck cancer patients represent a priority. Distinct from the articles [32] and guidelines [33] published during the first months of the pandemic, this review presenting the "COVID-19 organization protocol" of the research hospital "Casa Sollievo della Sofferenza" offers a different and enduring perspective accompanied by a new awareness of the disease and a gradual return to elective procedures. The COVID-19 pandemic has undoubtedly only accelerated the process toward the health care system of the future, where telemedicine [34], artificial intelligence [35] and mobile applications [36] are going to have more and more use.

\footnotetext{
Abbreviations

COVID-19: Coronavirus Disease 2019; SARS-CoV-2: Severe acute respiratory syndrome coronavirus 2; SIOeChCF: Italian Society of Otolaryngology-Head and Neck Surgery; ORL: Otolaryngology; ENT: Ear, Nose and Throat; URTI: Upper respiratory tract infections; ORL-HNS: Otolaryngology-head and neck surgery; AGPs: Aerosol-generating procedures; YO-IFOS: Young Otolaryngologists-International Federation of Otolaryngologic Societies; ISS: Higher Institute of Health; Cl: Cochlear implantation/implant; PPE: Personal protective equipment; AJCC: American Joint Committee on Cancer; OPHL: Open partial horizontal laryngectomy; CT: Computed tomography; BAL: Bronchoalveolar lavage; FESS: Functional endoscopic sinus surgery; DCR: Dacryocystorhinostomy; TORS: Transoral Robotic Surgery;
}

OSAS: Obstructive sleep apnea syndrome; AHI: Apnea hypopnea index; ICU: Intensive care unit; AIOCC: Italian Association of Head and Neck Oncology

\section{Acknowledgements}

We would like to thank Dr. Meghan M. Hiss for her comments which greatly improved this manuscript.

\section{Authors' contributions}

All authors meet the ICMJE criteria: 1) Substantial contributions to the conception or design of the work; or the acquisition, analysis, or interpretation of data for the work; 2) Drafting the work or revising it critically for important intellectual content; 3) Final approval of the version to be published; 4) Agreement to be accountable for all aspects of the work in ensuring that questions related to the accuracy or integrity of any part of the work are appropriately investigated and resolved. FL and EMCT were specifically responsible for the conception and design of the work.

\section{Funding}

None.

Availability of data and materials

Supplementary data and material are available upon request to the corresponding author.

Ethics approval and consent to participate

Not applicable.

Consent for publication

Not applicable.

Competing interests

The authors disclose no conflicts of interest.

\section{Author details}

${ }^{1}$ Department of Maxillofacial Surgery and Otolaryngology, IRCCS Casa Sollievo della Sofferenza, Viale Cappuccini, 1, 71013 San Giovanni Rotondo (Foggia), Italy. ${ }^{2}$ Department of Otolaryngology- Head and Neck Surgery, University Hospital of Foggia, Foggia, Italy. ${ }^{3}$ Department of Surgical Sciences; Unit of Maxillofacial Surgery, University Hospital of Turin- Città della salute e delle scienze, Turin, Italy. ${ }^{4}$ Department of Neurosciences, Reproductive and Odontostomatological Sciences, Maxillofacial Surgery Unit Naples, University of Naples Federico II, Naples, Italy.

Received: 27 November 2020 Accepted: 28 January 2021

Published online: 05 February 2021

\section{References}

1. Barillari MR, Bastiani L, Lechien JR, et al. A Structural Equation Model to Examine the Clinical Features of Mild-to-Moderate Covid-19: A Multicenter Italian Study. J Med Virol. 2020:jmv.26354. https://doi.org/10.1002/jmv.26354.

2. Mannelli G, Ralli M, Bonali M, Capasso P, Guarino P, lannini V, Mevio N, Russo G, Scarpa A, Spinato G, Topazio D, Molteni G. Impact of COVID-19 pandemic on Italian Otolaryngology Units: a nationwide study. Acta Otorhinolaryngol Ital. 2020;40(5):325-31. https://doi.org/10.14639/0392100XN0832.

3. Gelardi M, lannuzzi L, Trecca EMC, Kim B, Quaranta NAA, Cassano M. COVID-19: what happened to all of the otolaryngology emergencies? Eur Arch Oto-Rhino-Laryngology. 2020;18. https://doi.org/10.1007/ s00405-020-06046-z.

4. De Felice F, Polimeni A, Tombolini V. The impact of coronavirus (COVID-19) on head and neck cancer patients' care. Radiother Oncol. 2020;147:84-5. https://doi.org/10.1016/j.radonc.2020.03.020.

5. Ansarin M. Surgical management of head and neck tumours during the SARS-CoV (COVID-19) pandemic. Acta Otorhinolaryngol Ital. 2020;40(2):87-9. https://doi.org/10.14639/0392-100X-N0783.

6. Vukkadala N, Qian ZJ, Holsinger FC, Patel ZM, Rosenthal E. COVID-19 and the otolaryngologist - preliminary evidence-based review. Laryngoscope. 2020. https://doi.org/10.1002/lary.28672. 
7. Huart C, Philpott C, Konstantinidis I, et al. Comparison of COVID-19 and common cold chemosensory dysfunction. Rhinology. 2020. https://doi.org/ 10.4193/Rhin20.251.

8. Niklassen AS, Draf J, Huart C, et al. COVID-19: Recovery from Chemosensory Dysfunction. A Multicentre study on Smell and Taste. Laryngoscope, 2021: lary.29383. doi:https://doi.org/10.1002/lary.29383

9. Lechien JR, Chiesa-Estomba CM, Cabaraux P, et al. Features of mild-tomoderate COVID-19 patients with dysphonia. J Voice. 2020. https://doi.org/ 10.1016/j.jvoice.2020.05.012

10. Castelnuovo P, Turri-Zanoni M, Karligkiotis $A$, et al. Skull-base surgery during the COVID-19 pandemic: the Italian Skull Base society recommendations. Int Forum Allergy Rhinol. 2020;10(8):963-7. https://doi.org/10.1002/alr.22596.

11. Pichi B, Mazzola F, Bonsembiante A, et al. CORONA-steps for tracheotomy in COVID-19 patients: a staff-safe method for airway management. Oral Oncol. 2020;105:104682. https://doi.org/10.1016/j.oraloncology.2020.104682.

12. Chen JX, Workman AD, Chari DA, et al. Demonstration and mitigation of aerosol and particle dispersion during Mastoidectomy relevant to the COVID-19 era. Otol Neurotol. 2020;41(9):1230-9. https://doi.org/10.1097/ MAO.0000000000002765.

13. Al Kawas S, Al-Rawi N, Talaat W, et al. Post COVID-19 lockdown: measures and practices for dental institutes. BMC Oral Health. 2020;20(1):291. https:// doi.org/10.1186/s12903-020-01281-6.

14. Sowerby $L$, Stephenson K, Dickie A, et al. International registry of otolaryngologist-head and neck surgeons with COVID-19. Int Forum Allergy Rhinol. 2020;(xxxx):1-8. https://doi.org/10.1002/alr.22677.

15. Allevi F, Dionisio A, Baciliero U, et al. Impact of COVID-19 epidemic on maxillofacial surgery in Italy. Br J Oral Maxillofac Surg. 2020;58(6):692-7. https://doi.org/10.1016/j.bjoms.2020.04.035.

16. Cooper N. The World's best hospitals 2020. 2020. Accessed 25 Sept 2020. https://www.newsweek.com/best-hospitals-2020/italy

17. Copelli C, Tewfik K, Cassano L, et al. Management of free flap failure in head and neck surgery. Acta Otorhinolaryngol Ital. 2017;37(5):387-92. https://doi. org/10.14639/0392-100X-1376.

18. Kozin ED, Remenschneider AK, Blevins NH, et al. American Neurotology Society, American Otological Society, and American Academy of otolaryngology - head and Neck Foundation guide to enhance Otologic and Neurotologic care during the COVID-19 pandemic. Otol Neurotol. 2020; 41(9):1163-74. https://doi.org/10.1097/MAO.0000000000002868.

19. De Virgilio A, Pellini R, Mercante G, Ferreli F, Petruzzi G, Spriano G. Who should perform the rhinopharyngeal swab in COVID-19 positive patients? Head Neck. 2020;42(6):1250-1. https://doi.org/10.1002/hed.26194.

20. Società Italiana di Otorinolaringoiatria $(\mathrm{SIO})$. Tracheostomy during the COVID-19 pandemic [La tracheostomia in pazienti affetti da COVID-19]. 2020. https://www.sioechcf.it/riassunto-linee-guida-la-tracheostomia-inpazienti-affetti-da-covid-19/. Accessed 25 March 2020.

21. Società Italiana di Otorinolaringoiatria (SIO). Surgical activity during the COVID-19 pandemic [Attività chirurgica ORL]. 2020. https://www.sioechcf.it/ attivita-chirurgica-orl/. Accessed 28 March 2020.

22. Silverman DA, Lin C, Tamaki A, et al. Respiratory and pulmonary complications in head and neck cancer patients: evidence-based review for the COVID-19 era. Head Neck. 2020;42(6):1218-26. https://doi.org/10.1002/ hed.26217.

23. Piazza C, Filauro M, Dikkers FG, et al. Long-term intubation and high rate of tracheostomy in COVID-19 patients might determine an unprecedented increase of airway stenoses: a call to action from the European laryngological society. Eur Arch Oto-Rhino-Laryngology. 2020. https://doi. org/10.1007/s00405-020-06112-6.

24. Centers for Disease Control and Prevention. Considerations for Alternate Care Sites. 2020. https://www.cdc.gov/coronavirus/2019-ncov/hcp/ alternative-care-sites.html. Accessed 24 April 2020

25. Società Italiana di Otorinolaringoiatria $(\mathrm{SIO})$. Outpatient clinic during the COVID-19 pandemic [Accesso all'ambulatorio ORL in corso di pandemia da SARS-COV-2]. 2020. https://www.sioechcf.it/wp-content/uploads/2020/03/ Accesso-allambulatorio-ORL-in-corso-di-pandemia-da-SARS-COV-2.pdf. Accessed 26 March 2020.

26. Murr AT, Lenze NR, Gelpi MW, et al. Quantification of Aerosol Concentrations During Endonasal Instrumentation in the Clinic Setting. Laryngoscope.2020:lary.29122. doi:https://doi.org/10.1002/lary.29122

27. Mahoney MF. Telehealth, telemedicine, and related technologic platforms. J Wound, Ostomy Cont Nurs. 2020:47(5):439-44. https://doi.org/10.1097/WON. 0000000000000694.
28. Wasowski A, Skarzynski PH, Lorens A, Obrycka A, Walkowiak A, Bruski L. Remote Fitting of Cochlear Implant System. Cochlear Implants Int. 2010; 11(sup1):489-92. https://doi.org/10.1179/146701010X12671177318105.

29. AlOCC Al di OC-C. Make Sense Campaign. 2020. https://www. tienilatestasulcollo.aiocc.it/

30. Magaldi L, Salzo AE, Trecca EMC, lannuzzi L, Fortunato F, Cassano M. The importance of head and neck counselling in the COVID-19 era. Acta Otorhinolaryngol Ital. 2020:1-3. https://doi.org/10.14639/0392-100X-N0941.

31. Remuzzi A, Remuzzi G. COVID-19 and Italy: what next? Lancet. 2020; 395(10231):1225-8. https://doi.org/10.1016/S0140-6736(20)30627-9.

32. De Bernardi F, Turri-Zanoni M, Battaglia P, Castelnuovo P. How to Reorganize an Ear, Nose, and Throat Outpatient Service During the COVID -19 Outbreak: Report From Northern Italy. Laryngoscope 2020:lary.28716. doi:https://doi.org/10.1002/lary.28716

33. Guidance for ENT during the COVID-19 pandemic. Published 2020. Accessed March 23, 2020. https://www.entuk.org/sites/default/files/files/ Guidance for ENT during the COVID-19 pandemic.pdf.

34. Lambertoni A, Gravante G, Battaglia P, Turri-Zanoni M, Castelnuovo P, Karligkiotis A. A "Forward Triage" Model in Telemedicine for Head and Neck Oncological Patients During the COVID-19 Era. Ear, Nose Throat J. 2020: 014556132094899. doi:https://doi.org/10.1177/0145561320948995

35. Tama BA, Kim DH, Kim G, Lee S, Kim SW. Recent Advances in the Application of Artificial Intelligence in Otorhinolaryngology-Head and Neck Surgery. Clin Exp Otorhinolaryngol. 2020. https://doi.org/10.21053/ceo.2020. 00654.

36. Trecca EMC, Lonigro A, Gelardi M, Kim B, Cassano M. Mobile Applications in Otolaryngology: A Systematic Review of the Literature, Apple App Store and the Google Play Store. Ann Otol Rhinol Laryngol 2020:000348942094035. doi:https://doi.org/10.1177/0003489420940350

\section{Publisher's Note}

Springer Nature remains neutral with regard to jurisdictional claims in published maps and institutional affiliations.

Ready to submit your research? Choose BMC and benefit from:

- fast, convenient online submission

- thorough peer review by experienced researchers in your field

- rapid publication on acceptance

- support for research data, including large and complex data types

- gold Open Access which fosters wider collaboration and increased citations

- maximum visibility for your research: over $100 \mathrm{M}$ website views per year

At BMC, research is always in progress.

Learn more biomedcentral.com/submissions 